\title{
Contributing Factors to Implementation Gaps in Rehabilitation Services in South Africa: Perspectives from health facility-level Rehabilitation Clinician-Managers
}

Qhayiya Magaqa ( $\sim$ qhayiya.magaqa@ndm.ox.ac.uk)

\section{Research}

Keywords: rehabilitation services, policy implementation

Posted Date: March 26th, 2021

DOI: https://doi.org/10.21203/rs.3.rs-357899/v1

License: (c) (1) This work is licensed under a Creative Commons Attribution 4.0 International License. Read Full License 


\section{Abstract}

Previous studies have examined health policy implementation globally. However, few studies have examined policy implementation as it relates to rehabilitation services. This study aimed to report on the factors which may be contributing to policy implementation gaps in rehabilitation services in a rural district of South Africa. Qualitative semi-structured interviews were conducted with rehabilitation providers who have both clinical and managerial duties in health facilities. The framework method was used to guide analysis. Findings suggest that gaps between rehabilitation policy and the available services may be attributable to a lack of systems hardware and the presence of maladaptive systems software. Additionally, the limited ability of rehabilitation managers to exercise their power to plan and organise rehabilitation services constrained policy implementation. The implications of this work point to the need to better include rehabilitation managers in priority setting and resource allocation processes at the level of the health facility.

\section{Introduction}

Various studies in low- and middle-income countries (LMICs) and high-income countries (HIC) have explored the implementation of policies and have shown that the implementation of policies by stakeholders at the coalface of service provision is complex and dynamic. These studies have examined the people involved in the process of implementation, as well as how their roles, relationships and views influence implementation (Ditlopo et al., 2015; Razavi et al., 2019; Urquhart et al., 2019; Lall et al., 2020; Parashar et al., 2020). Other studies have reported on how resource availability enhances or limits policy implementation at the health facility level (Gilson et al., 2017; Waithaka et al., 2018; Hlayisi and Ramma, 2019; Muthathi, Levin and Rispel, 2019). These studies have described the processes involved in determining need for such resources, the allocation of resources and purchasing arrangements of obtaining resources. Still, other studies have focussed on how the evaluation of policy goals including accountability monitoring and the required data systems, also inform whether and how policy aspirations are achieved (Gilson et al., 2017; Suchman et al., 2020).

However, with the exception of Hlayisa and Ramma (2019) whose study focuses on South Africa, none of these studies have explored policy implementation as it relates to rehabilitation services. Rehabilitation services serve an important role in reducing the burden of morbidity and improving the functioning of individuals, yet have traditionally been underprioritized within health systems as evidenced by persisting unmet needs for rehabilitation services (Bernhardt et al., 2020; Cieza et al., 2020). Given that South Africa's UHC plan includes rehabilitation services as part of the service package which will be made available to the population, understanding the current rehabilitation implementation challenges may support successful implementation of rehabilitation-inclusive health services. Therefore, this research aimed to explore the multiple factors which may be contributing to the implementation gap with the following research question: Why is there an implementation gap between what South Africa's rehabilitation policies state and the available rehabilitation services in health facilities in OR Tambo district? 


\section{Materials And Methods}

Study Site

The study was situated in OR Tambo district, a rural district of the Eastern Cape province in South Africa. The district has a population of 1.4 million (O.R. Tambo District Municipality, 2017). The primary health care service delivery platform consists of nine district hospitals and a total of 146 clinics and community health centres (Department of Health, 2019; Massyn et al., 2020). Regarding the provision of rehabilitation services in OR Tambo district, 2020 estimates reported that per 100000 population, there were 2.1 physiotherapists and 1.5 physiotherapists (Massyn et al., 2020). In absolute numbers, there were 30 rehabilitation providers employed across the nine district hospitals in OR Tambo in 2019 (OR Tambo district office, personal communication). Rehabilitation services are provided in all district hospitals by employed rehabilitation providers. Since the clinics and community health centres in the district do not employ rehabilitation providers, outreach services are conducted by the district hospitals' rehabilitation teams.

\section{Data Collection}

This study formed part of a larger study examining rehabilitation policy and rehabilitation service capacity in district hospitals in OR Tambo district. The research was a case study of health-facility rehabilitation managers in OR Tambo district (Fraser and Mays, 2020). The study adopted a social constructivist paradigm since various stakeholders involved in rehabilitations service delivery in the district may offer differing perspectives regarding policy implementation (Neuman, 2014). The healthfacility rehabilitation managers were selected because of their frontline experience of implementing rehabilitation policy. The sampling strategy involved convenience sampling due to resignations and leaves of absence (Neuman, 2014; Hinton and Ryan, 2020). In each health facility (district hospital), rehabilitation providers who were formally recognised in their respective health facilities as being responsible for the planning, organisation and delivery of rehabilitation services were invited to participate in the study. Additionally, in order to explore a case of positive deviance as described by Neuman (2014), a senior manager in one health facility who oversees the clinical team including the medical and broader allied teams was also recruited. This health facility has a reputation of offering a well-functioning rehabilitation service.

After written consent was obtained from the recruited rehabilitation managers, the author collected the data using semi-structured interviews informed by the World Health Organisation's (WHO) health system components (WHO, 2010). The interview guide (supplementary 1 ) which was developed for this study contained guiding questions related to leadership and governance, rehabilitation service delivery, rehabilitation workforce, financing for rehabilitation services, assistive technologies, and health information systems. Appropriate prompts such as "Could you tell me more about...", "What do you mean by...", "Could you clarify who..." and "Am I correct in saying that what you have said is..." were used to gain clarity or to delve deeper into the insights raised during the interviews. Each interview was conducted in English and took approximately one hour, although the interviews in which more than one participant was 
included took longer. The interviews were then audio-recorded and transcribed by the author in preparation for analysis in NVivo 12 (QSR International, 2017).

Analysis

The Framework Method was used to analyse the interview transcripts given the rehabilitation policy review and health facility assessment findings (as examined in another part of this research) (Gale et al., 2013; Pope, Ziebland and Mays, 2020). This type of analysis has previously been used in policy analysis and lends itself to the exploration of how rehabilitation policy is implemented at the health facility level in district hospitals by rehabilitation managers. This enabled a focussed approach to the policy and health system areas identified a-priori. The framework which conceptualises the health system as having both systems hardware and software was adopted to guide the analysis (Sheikh et al., 2011). While literature amongst rehabilitation managers is limited, research amongst nurses and physicians who act as managers of services, and other frontline workers facilitated the a-priori formulation of codes, categories and themes (Ditlopo et al., 2011, 2015; Nzinga, McGivern and English, 2018; Rwafa-Ponela et al., 2020). Where themes previously not mentioned in the literature arose, these were coded and categorised. Ethical approval to conduct the study was obtained from Oxford University (OxTREC 513 - 19), Walter Sisulu University Human Research Committee (042/2019), and the Eastern Cape provincial Department of Health (EC_201907_001).

Reflecting on Positionality

As a physiotherapist who worked previously worked in OR Tambo district, I was aware that I have my own views and perspectives about rehabilitation services in OR Tambo district. Thus, I had an insider perspective with regards to rehabilitation service delivery in the district and the providers from one health facility. However, I also had an outsider perspective because it had been several years since I had left my clinical role. Additionally, I did not have experience of the service provision of the other eight health facilities included, which is why it was important to meet them informally prior to data collection. Having said that, I was intentional about making the participants aware of where I had worked previously. My background and experience influenced my data collection in that I tended to be careful to not over-step or abuse boundaries. In practice what that meant was I only went to the areas where the interview was taking place and did not enter any of the wards or disturb patient consultations. During the interview, I checked with participants to clarify whether what I was comprehending was true to the meaning which they were attaching to their words, facial expressions, silences or body movements. With regards to the analysis, and in particular to my interpretation of the themes, I was aware that I needed to constantly refer to the themes as they appeared in the transcripts even if they indicated an experience that was different to my time of working in the district. Having said that, my aim was to represent the experiences of the participants.

\section{Results}

Characteristics of Respondents 
The respondents represented seven of the nine health facilities in OR Tambo district. Amongst these respondents, half of them were community service officers, which are rehabilitation providers in their first year of work after their degree qualification (Table 1). The remainder were permanent providers, most of whom had three years or less of occupying their clinician-manager role. Regarding the professions of the respondents, their characteristics are listed in Table 2.

Table 1

Respondents according to professional level

\begin{tabular}{|ll|}
\hline Professional Level & Respondents $(n=12)$ \\
\hline Community service officer & 6 \\
\hline Permanent staff & 6 \\
\hline
\end{tabular}

Table 2

Respondents according to type of provider

\begin{tabular}{|ll|}
\hline Profession & Respondents $(n=12)$ \\
\hline Occupational therapist & 4 \\
\hline Physiotherapist & 4 \\
\hline Audiologist & 2 \\
\hline Speech and Language therapist & 1 \\
\hline Medical doctor & 1 \\
\hline
\end{tabular}

The findings from the interviews with the rehabilitation managers will first be reported according to components of the rehabilitation service namely leadership and governance, financing, assistive technology, service delivery and rehabilitation workforce and health information systems. Thereafter, the participants' experiences and perspectives as they relate to relationships and power, ideas and interests, and values and norms will be reported.

Systems Hardware

\section{Leadership and Governance}

Findings under the theme of leadership and governance revealed that the managers of the rehabilitation services in health facilities were not formally employed as managers but were accepted and functioned as such in the health facilities. In other words, they were employed as clinicians and given additional managerial responsibilities. Moreover, most of the rehabilitation managers landed in these clinicianmanager roles unexpectedly as opposed to through applying for them.

"Yes, I am the person who is in charge of the physiotherapy department in the whole hospital. So even though I am a comm serve, but I ended up doing more. Like maybe I'm supposed to be supervised by the 
senior physio but that's not happening because I'm the only one. So anything related to physio, it's me." (RM 5, Community Service level, physiotherapist, HF 6)

An important aspect of their roles as clinician-managers was to provide a service that aligned with policy guidance from the Department of Health. However, the participants' responses emphasised that the implementation of policies was constrained by factors related to the rehabilitation policies themselves, resource unavailability, governance tensions and exclusion from priority setting and resource allocation processes relevant to rehabilitation services at the health facility.

"It's not always easy to because those policies are just looking at all hospitals whether district or regional. Sometimes you look and see that you are limited because whether it's the environment or it's the equipment that you cannot achieve some of the goals that you should have achieved... So even with the hearing aids, I have a limited stock so the cut doesn't cover the whole of my patients. Even with the new policy that came out- that we're supposed to fit two hearing aids for kiddies and one hearing aid for an adult-but when I look at the stock that we get, if I were to fit continuously two for kiddies, I would run out of stock very soon, whereas other hospitals wouldn't. (RM 3c, Permanent, audiologist, HF 3)

\section{Financing}

Findings under the theme of financing were directly related to the theme of leadership and governance. Specifically, the responses pointed to low levels of participation by the rehabilitation managers in the annual health facility budget allocation processes. Additionally, the budget allocation for rehabilitation services was unpredictable making it difficult for the rehabilitation managers to anticipate and plan for resources in the following financial period. However, despite these challenges, an instance of leveraging routinely collected data in one health facility resulted in an increased budget allocation for rehabilitation services in the proceeding financial year.

"We would love to be included in budget allocation, you know, because they don't know what we need specifically. So that's what I am referring to because that's our main issue. So they (senior management) meet up and say that we've been given this amount for the financial year. "We are allocating R30 000 to OT. We are not allocating money to physiotherapy". Which is something that happened in 2019. So if we would be included in those meetings we would voice out our needs and then they can make decisions with us and not for us, do you understand?" (RM 2, Permanent, occupational therapist, HF 2)

\section{Assistive Technology}

Again, there were strong connections between financing for rehabilitation services and access to assistive technology. The impact of the unpredictable nature of the budget allocation at the level of the health facility was directly related to what assistive devices and consumables could be procured and provided through the health facility. The result of an unpredictable budget was a limited supply of assistive devices and consumables which could be prescribed to patients or used during rehabilitation treatment sessions with patients. Not only was the budget unpredictable but it was also not amenable to change, as evidenced by the quote from one rehabilitation manager: 
"And then they have a budget where they've got items on the list and if your thing is not literally named by name, it's not an item. And I'm yet to figure out how to get a new item on the list." (RM 3a, permanent, occupational therapist, HF 3)

In light of these constraints, a few rehabilitation managers overcame these constraints by conducting additional fundraising activities and leveraging the bureaucratic process to fit the rehabilitation department's needs.

"So we would privately fundraise to buy things which is unheard of, and we are fortunate to have an NGO connected that has all the correct documentation that they can donate to us so it's approved... So, I think, that is very good and it helps a lot." (RM 3a, permanent, occupational therapist, HF 3)

"So even though the budget says OT consumables, we use that budget for both OT and physio." (RM 6, community service, physiotherapist, HF 7)

There was also a strong awareness amongst the participants that the availability of assistive devices and consumables directly impacted the rehabilitation managers' capacity to deliver rehabilitation services. Thus, highlighting the interaction between the components of assistive technology, consumables and rehabilitation service delivery.

\section{Service delivery and Rehabilitation Workforce}

Findings within this theme uncovered factors which may be affecting policy implementation in accessibility, rehabilitation service capacity and the rehabilitation workforce. Firstly, the provision of outreach services to surrounding clinics was constrained by a lack of available transport meaning that rehabilitation service provision at these clinics was intermittent. In a few health facilities, low numbers of rehabilitation providers was cited as a major constraining factor to providing outreach services since it would mean the closing of the service for the day.

"We don't have enough vehicles" (RM 7, community service level, audiologist, HF 9)

"The problem is, no there are no other ways. They have to come to the hospital, reason being I can't do the outreach because that would mean if I go to the nearest clinic, we are going to close the hospital (physiotherapy department). So people who are here won't be able to be seen because I'm the only one, you know what I'm saying?" (RM 5, community service, physiotherapist, HF 6)

Secondly, sub-optimal treatment spaces compromised the accessibility of rehabilitation departments in two health facilities and impacted how many patients could be attended to at a particular time.

"We need a space... Look at this place, it's too small: two people, two departments. It's supposed to be just one testing room and then we have a separate office. Because how we operate now, if I have a patient, she has to wait. If she has a patient, I must wait." (RM 3c, permanent, audiologist, HF 3)

\section{Health Information Systems}


Findings under the theme of health information systems pointed to data as an important contributor to the implementation gaps in rehabilitation service capacity and the rehabilitation workforce. Firstly, workforce planning tools for rehabilitation were said to be unheard of by the rehabilitation managers. Therefore, they could not anticipate the supply of the rehabilitation workforce and plan the service accordingly.

"It's very sporadic. Now you get two, next year you get none, now this hospital has no therapists, now suddenly they have comm serves for the first time in how many years." (RM 3a, permanent, occupational therapist, HF 3)

Secondly, the routinely collected service provision data was an inadequate representation of the rehabilitation service provided in practice. Moreover, the sparse data that was collected was not used to inform improvements in the service.

"The other thing is that wheelchairs are so few and far in between than the other things that we do. A wheelchair is merely a tool for the patient. It's not the biggest part of rehab. And yet that is what we are based on...So (we should be) including more detail rather than "did they need a wheelchair or not". So if they did need an assistive device, was it maybe a washing board or a splint. There is nowhere that splints are mentioned where splints are so functional and needed for so many different patients." (RM 6, community service, occupational therapist, HF 7)

Systems Software

\section{Relationships and Power}

The areas where the rehabilitation managers perceived themselves to have limited or no power were in the areas of leadership and governance, and financing in health facilities. In all the health facilities the respondents functioned as managers of rehabilitation services in their respective health facilities while being officially employed as clinician rehabilitation providers. In other words, their employment records documented them as primarily clinicians rather than employed in "Chief" posts such as Chief Occupational Therapist. In practice, this meant that while the respondents had to fulfil all the expectations associated with being a manager, they did not really have the power to make important decisions related to the planning, organising and delivery of rehabilitation services. What further compounded this perceived lack of power or agency was how unprepared to manage they felt, especially because they had not chosen the role for themselves. Moreover, rehabilitation managers often did not participate in health facility level resource allocation discussions and decisions, resulting in budget allocations which were not responsive to the rehabilitation needs of the catchment area. This contributed to the rehabilitation managers' reduced ability to act to organise and deliver rehabilitation services in accordance with the relevant Department of Health policies.

\section{Ideas and Interests}


Interestingly, the perceived lack of power that the rehabilitation managers felt in the areas of leadership and governance, and financing led in some cases to innovative ideas in the areas of access to medical technologies and rehabilitation service delivery. One rehabilitation manager from HF 3 reported conducting fundraising activities in order to compensate for the lack of a consumables budget. While in HF 2, one rehabilitation manager leveraged the wheelchair service records to advocate for additional health facility funding for wheelchairs in the proceeding financial year.

"We gave them those (DHIS) stats. We told them our patients wait two to three years to get their wheelchairs. So in the interim there is something we must do. So last year, the comm serve and I attended a lot of those meetings and we told them that "we need wheelchairs, we need wheelchairs, we need wheelchairs". So last year we got R30 000 and they added another R30 000 towards the end of the year. Then this year it was R70 000." (RM 1, permanent, occupational therapist, HF 1)

Regarding service delivery and expanding coverage, one rehabilitation manager from HF 7 reported providing speech and language therapy outreach services to two nearby district hospitals which did not offer speech and language therapy services, which was unusual as outreach services were usually conducted at clinics.

\section{Values and Norms}

The reasons why ideas and innovation were sparked in these areas in particular rather than other areas may be related to the fact that the areas of access to medical technologies and service delivery more readily aligned with the clinical training of the rehabilitation managers, and thus they had more experience in enacting roles with reference to these areas. In light of the constraints, one rehabilitation manager from HF 9 noted the need to have to draw from internal personal resources to overcome the constraints. In particular, they felt that one needed to be a "strong hearted person" rather than "fainthearted" (RM 7, community service level, audiologist, HF 9). Indeed, many of the rehabilitation managers expressed having values which anchored their practice. These values were mostly personal although there were some which were institutional. The values included treating everyone like family, including the patient, and aiming to "leave a good mark on something" (RM1, permanent, occupational therapist, HF 1). Similarly, another rehabilitation manager from HF 6 said it helped them to remember that every patient could have been their mother, and was therefore worthy of being treated well (RM5, community service level, physiotherapist, HF 6).

Two rehabilitation managers from HF 3 mentioned institutional values that were communicated throughout the whole health facility. These institutional values were "to provide quality care and to provide gold standard care in a rural setting" despite the limited resources (RM 3a, permanent, occupational therapist, HF 3 and RM3c, permanent, audiologist, HF 3). The institutional values in HF 3 seemed to enable an establishment of practiced norms in a way that did not happen in other health facilities. Teamwork, and in particular inter-disciplinary teamwork seemed to be an important aspect of enacting what "quality service" looked like. 


\section{Discussion}

The findings from interviews with clinician-managers of rehabilitation services in health facilities in OR Tambo district suggest that the gap between rehabilitation policies and their implementation may be explained by a lack of the systems hardware which is necessary for the provision of rehabilitation services. This lack of systems hardware co-existed alongside systems software which were either adaptive and in line with policy aspirations, or maladaptive and not aligned with policy aspirations.

The mechanism by which a lack of systems hardware contributed to the implementation gap is through limited financial resources for procuring assistive technologies and consumables, limited transport and workforce for outreach, and an unclear use of records to inform planning. This directly influenced the availability of components of the service. For example, a limited budget constrained the assistive devices which could be available at the health facility. In health facilities which did provide outreach services, a limited supply of health facility transport directly affected service coverage to clinics. Conversely, in health facilities that did not provide outreach services, limited rehabilitation staff members prevented service coverage to clinics. Lastly, while all health facilities reported submitting service records to the provincial rehabilitation forum, there was no reported evidence that changes were being instituted at the health facility level in response to the information generated by the service records.

Regarding the maladaptive software present across the health facilities, this also prevented rehabilitation policies being fully implemented. The distinction between adaptive and maladaptive software is to emphasise that systems software is always present in systems but that it may either support (adaptive) or constrain (maladaptive) the implementation of policies. This maladaptive software in this study was most exemplified in the limited power of the rehabilitation managers. While the rehabilitation managers did have some power, it was not being exercised in a way which enabled the full implementation of policies at the health facility level. This is because how managers exercise their power directly impacts whether policy implementation is supported or constrained (Gilson, Schneider and Orgill, 2014). In the case of OR Tambo district, rehabilitation policy implementation was constrained by limitations in the power of the rehabilitation managers.

While there were examples of adaptive software, that is software which supports the implementation of policies, these examples arose in response to constraints in the systems hardware inside the health facility or across health facilities. For example, the rehabilitation managers who reported fundraising through the local NGO did so because there was no budget allocated at the health facility to procure consumables. Another example is that the rehabilitation managers who reported advocating for an increase in budget for wheelchairs did so because their wheelchair needs according to the waiting list were not being addressed. Lastly, the rehabilitation manager who provided outreach services to other district hospitals instead of clinics, did so because those two district hospitals did not have speech and language therapy providers. In each of these examples, the managers' reported actions ultimately resulted in the fulfilment of the relevant policy aspirations even though the way in which this was achieved was not as originally intended by the policies. 
Drawing from VeneKlasen and Miller's framework of expressions of power (Table 3), we see how power is central to the rehabilitation managers' experiences of implementing rehabilitation policies and relatedly, the gaps seen in their implementation in OR Tambo district (VeneKlasen and Miller, 2007). The rehabilitation managers may also be termed hybrid-managers since they had both clinical and managerial duties (McGivern et al., 2015). These rehabilitation managers were clinicians who had landed in their roles by default but their power was challenged by the lack of legitimacy surrounding their managerial roles given that their manager-clinician roles were not recognised by the Department of Health. Arguably, what may make the experience of rehabilitation managers particularly unique is their status within the medical hierarchy. Since medical doctors are often recognised and acknowledged as superior to other healthcare workers including by rehabilitation providers themselves, this relative status may account in part for why rehabilitation managers' participation was lacking in resource allocation procedures.

Table 3

How power is enacted

Expressions of Power (VeneKlasen and Miller, 2007)

Power over. Involves taking it from someone else and dominating and subjugating others.

Power with: Involves finding common ground among different interests and building collective strength. Includes mutual support, solidarity and collaboration.

Power to: Recognised the unique potential of every person to shape his or her life and world.

Power within: Recognised a persons' sense of self-worth and self-knowledge, and recognising individual differences while respecting others. Capacity to imagine and have hope, and affirms common human search for dignity and fulfilment.

This resulted in the rehabilitation managers not being able to leverage their technical expertise as rehabilitation experts to the same extent that medical doctors might leverage their clinical expertise to influence decisions regarding procurement of medicines at a health facility level (Barasa et al., 2016). Thus, the rehabilitation managers could not derive power from their professional status within the hierarchy because as professionals, they featured in the lower ranks within the hierarchy. The ability to leverage power is particularly important in environments such as OR Tambo district, where there are high needs, competing priorities and limited resources, as has also been found in district-level decision making amongst health stakeholders in Uganda (Razavi et al., 2019). Despite the hospital level as in OR Tambo, and the district level as in Uganda, operating with different resources and responsibilities, it is highly likely that influencing decision making by stakeholders through leveraging different types of power happens at all levels of the health system.

Despite this limited power, rehabilitation managers were not completely without power. Instead, they harnessed and exercised power with. Innovation arising from constraints was an expression of exercising power with. It was the extensive experience and power over the rehabilitation team, coupled with a strong pursuit of collaboration as a core ethos in HF 3 that resulted in a beneficial relationship for rehabilitation 
managers between the NGO set up to support the hospital, and the fundraising activities. This contrasts the findings of another study in which a reduced sense of power in decision making in hospitals of middle-level managers resulted in a culture of feigned compliance rather than innovation as demonstrated in the current study's findings (Barasa et al., 2017). In another study, health facility managers collaborated to re-introduce user fees while waiting for the government to make health facility funds available (Gilson et al., 2017). This is interesting because it suggests that in the presence of resource scarcity to provide services, managers will either externally appear compliant with policy aspirations or they will innovate. Either way, in both instances while it may appear that policy aspirations are being met overall, the current opportunity costs may in the longer term negatively impact service delivery and the population. This inevitable practice of power, whether formal or informal, is something that has also been found amongst stakeholders involved in the implementation of a national community health worker policy in South Africa (Lehmann and Gilson, 2013). Indeed, rehabilitation managers in OR Tambo district did not have formal power derived from their professional level, but they did exercise the agency that they had to implement rehabilitation service delivery according to their own understandings of their roles and of the service.

Since Sheikh et al's (2011) systems hardware and software framework looks at systems holistically, it does not explain why one health facility consistently fared better than the others. What made HF $3 \mathrm{a}$ health facility which, although it did have implementation gaps but fared better than the others, is that the rehabilitation managers benefited from more sources of power available to them compared to all the other health facilities. The clinical manager derived power from the combination of being a medical doctor, his position of authority over the health facility's clinical team including the rehabilitation team and being employed in HF 3 for over a decade. This clinical manager leveraged his power and experience as a senior manager to support and mentor the rehabilitation managers in that health facility. VeneKlasen and Miller identify power over as an inherently negative concept suggesting domination and oppression. However, arguably in the case of HF 3 , the clinical manager exercised his power over not for subjugation but for the benefit of the rehabilitation service. It could also be argued that the rehabilitation managers in all the health facilities needed a space to express power over, that is to have control over human and material resources which comprised rehabilitation service capacity in their health facilities. The rehabilitation managers needed to have power over with the intention to better plan and deliver services, as would be expected of any healthcare manager.

The managers in the current study reported that they had little or no access to policies similar to what has previously been found amongst rehabilitation managers across national and sub-national healthcare levels in South Africa (Dayal, 2010). However, where participants did report having access to policies, implementation was constrained by a limited sense of power to drive meaningful change and a lack of the necessary resources for service delivery. In other words, their limited ability to exercise power constrained policy implementation, resulting in a gap between policy and available rehabilitation services (Gilson, Schneider and Orgill, 2014). The participants in the current study were not officially employed as rehabilitation managers. While they had the power and responsibility to provide an account of rehabilitation services to the senior health facility managers and provincial managers, they did not feel 
they had the power to make meaningful changes to the service according to their professional experience and perspectives. This may be described as having a limited decision space where decision space refers to how much practical authority and choice an individual is formally or informally afforded in an organisation (Bossert 1998 in Muthathi, Levin and Rispel, 2019). This limited decision space, attributable to limited participation in budget processes, has also been reported amongst health care facility managers in South Africa regarding vital components of the service such as the availability of essential medication and resuscitation equipment (Muthathi, Levin and Rispel, 2019).

\section{Conclusion}

The findings from this study support the growing evidence base that emphasises the crucial role of frontline managers, and in this case rehabilitation managers, in health policy implementation. The interplay between limitations in the available systems hardware and the presence of maladaptive systems software, coupled with the limited power of rehabilitation providers may explain the gap between rehabilitation policies, and the available rehabilitation services across health facilities in OR Tambo district. This study highlights the significant role of health facility rehabilitation managers in fulfilling rehabilitation policy mandates. Specifically, involving rehabilitation managers in resource allocation and priority setting processes may be an important step towards supporting the successful planning, organisation and delivery of rehabilitation services in health facilities.

A major strength of this study is the exploration of multiple components of the rehabilitation services instead of a single component, thus giving a more holistic view with the related interactions between components. However, this study also has limitations. Firstly, the voices of those actually involved in the resource allocation process at the health facility level were not included. Therefore, while the impact of budget allocation process is described in this study, the innerworkings of why and how that process takes place are not described in this work. Further work should also explore the roles and perspectives of senior managers at health facility and provincial level who have a role in organising and planning rehabilitation services. Secondly, these findings represent a discreet period amongst a particular group of managers. Thus, changes in the organisation and delivery of rehabilitation services over time was not observed or reported. This is of particular importance because of the high turnover of rehabilitation providers in rural health facilities. Further research should employ ethnographic approaches with longer engagement and observation of rehabilitation managers in order to better capture their practice as managers in context.

\section{Declarations}

\section{Competing Interests}

The author declares that there are no competing interests.

\section{Funding}


This research was funded by the Oppenheimer Memorial Trust, Africa Oxford Initiative and Green Templeton College, Oxford University.

\section{Acknowledgements}

I would like to thank Associate Professor Proochista Ariana and Dr Sarah Polack for their support and guidance during the research process.

\section{References}

Barasa, E. W. et al. (2016) 'The influence of power and actor relations on priority setting and resource allocation practices at the hospital level in Kenya: a case study', BMC Health Services Research. BioMed Central, 16(1), pp. 1-13. doi: 10.1186/s12913-016-1796-5.

Barasa, E. W. et al. (2017) 'Hospitals as complex adaptive systems: A case study of factors influencing priority setting practices at the hospital level in Kenya', Social Science \& Medicine, 174, pp. 104-112. doi: 10.1016/j.socscimed.2016.12.026.

Bernhardt, J. et al. (2020) Stroke 3 Stroke rehabilitation in low-income and middle-income countries: a call to action, The Lancet. doi: 10.1016/S0140-6736(20)31313-1.

Cieza, A. et al. (2020) 'Global estimates of the need for rehabilitation based on the Global Burden of Disease study 2019: a Systematic Analysis for the Global Burden of Disease Study 2019', The Lancet. The Author(s). Published by Elsevier Ltd. This is an Open Access article published under the CC BY-NC-ND 3.0, 6736(20). doi: 10.1016/S0140-6736(20)32340-0.

Dayal, H. (2010) 'Provision of rehabilitation services within the District Health System - the experience of Rehabilitation Managers in facilitating this right for People with Disabilities', SA Journal of Occupational Therapy South African Journal of Occupational Therapy, 40(1). Available at:

http://www.scielo.org.za/pdf/sajot/v40n1/06.pdf (Accessed: 23 November 2017).

Department of Health (2019) Primary Health Care Facilities and Services. Available at: https://www.healthestablishments.org.za/Home/Facility (Accessed: 27 March 2020).

Ditlopo, P. et al. (2011) 'Analyzing the implementation of the rural allowance in hospitals in North West Province, South Africa', Journal of Public Health Policy, 32(S1), pp. S80-S93. doi: 10.1057/.jphp.2011.28.

Ditlopo, P. et al. (2015) 'Contestations and complexities of nurses' participation in policy-making in South Africa', Global Health Action. Taylor and Francis Ltd., 8, pp. 26-34. doi: 10.3402/gha.v7.25327.

Fraser, A. and Mays, N. (2020) 'Case Studies', in Pope, C. and Mays, N. (eds) Qualitative Research in Health Care. Fourth Edition. Wiley and Sons Ltd, pp. 181-191. doi: 10.1002/9781119410867.ch13. 
Gale, N. K. et al. (2013) 'Using the framework method for the analysis of qualitative data in multidisciplinary health research', BMC Medical Research Methodology. BioMed Central, 13(1), p. 117. doi: 10.1186/1471-2288-13-117.

Gilson, L. et al. (2017) 'Everyday resilience in district health systems: Emerging insights from the front lines in Kenya and South Africa', BMJ Global Health. BMJ Publishing Group, 2(2). doi: 10.1136/bmjgh2016-000224.

Gilson, L., Schneider, H. and Orgill, M. (2014) 'Practice and power: a review and interpretive synthesis focused on the exercise of discretionary power in policy implementation by front-line providers and managers', Health Policy and Planning, 29(suppl 3), pp. iii51-iii69. doi: 10.1093/heapol/czu098.

Hinton, L. and Ryan, S. (2020) 'Interviews', in Qualitative Research in Health Care. Wiley, pp. 43-55. doi: 10.1002/9781119410867.ch4.

Hlayisi, V. G. and Ramma, L. (2019) 'Rehabilitation for disabling hearing loss: evaluating the need relative to provision of hearing aids in the public health care system', Disability and Rehabilitation. Taylor and Francis Ltd, 41(22), pp. 2704-2707. doi: 10.1080/09638288.2018.1473507.

Lall, D. et al. (2020) 'Team-based Primary Health Care for Non-communicable Diseases: Complexities in South India', Health Policy And Planning, 35(2), pp. 22-34. doi: 10.1093/heapol/czaa121.

Lehmann, U. and Gilson, L. (2013) 'Actor interfaces and practices of power in a community health worker programme: A South African study of unintended policy outcomes', Health Policy and Planning. Health Policy Plan, 28(4), pp. 358-366. doi: 10.1093/heapol/czs066.

Massyn, N. et al. (2020) District Health Barometer: District Health Profiles 2018/19.

McGivern, G. et al. (2015) 'Hybrid Manager-Professional' Identity Work: The Maintenance and Hybridization of Medical Professionalism in Managerial Contexts', Public Administration, 93(2), pp. 412432. doi: 10.1111/padm.12119.

Muthathi, I. S., Levin, J. and Rispel, L. C. (2019) 'Decision space and participation of primary healthcare facility managers in the Ideal Clinic Realisation and Maintenance programme in two South African provinces', Health Policy and Planning, pp. 1-11. doi: 10.1093/heapol/czz166.

Neuman, L. W. (2014) Social Research Methods: Qualitative and Quantitative Approaches. Seventh. Essex: Pearson Education Limited. Available at: www.pearsoned.co.uk (Accessed: 28 November 2018).

Nzinga, J., McGivern, G. and English, M. (2018) 'Examining clinical leadership in Kenyan public hospitals through the distributed leadership lens', Health Policy and Planning, 33(suppl_2), pp. ii27-ii34. doi: 10.1093/heapol/czx167. 
O.R. Tambo District Municipality (2017) Integrated Development Plan, 2017-2022. doi:

10.1017/CB09781107415324.004.

Parashar, R. et al. (2020) 'Unpacking the Implementation Blackbox using 'actor interface analysis': How did Actor Relations and Practices of Power influence Delivery of a Free Entitlement Health Policy in India?', Health Policy And Planning, 35(2), pp. 74-83. doi: 10.1093/heapol/czaa125.

Pope, C., Ziebland, S. and Mays, N. (2020) 'Analysis', in Pope, C. and Mays, N. (eds) Qualitative Research in Health Care. Fourth. John Wiley and Sons Ltd, pp. 111-133.

QSR International (2017) 'Nvivo 11'. Doncaster: QSR International Pty Ltd.

Razavi, S. D. et al. (2019) 'Who is in and who is out? A qualitative analysis of stakeholder participation in priority setting for health in three districts in Uganda', Health Policy and Planning, 34(5), pp. 358-369. doi: 10.1093/heapol/czz049.

Rwafa-Ponela, T. et al. (2020) 'Implementing without guidelines, learning at the coalface: A case study of health promoters in an era of community health workers in South Africa', Health Research Policy and Systems. BioMed Central Ltd., 18(1). doi: 10.1186/s12961-020-00561-5.

Sheikh, K. et al. (2011) 'Building the Field of Health Policy and Systems Research: Framing the Questions', PLoS Medicine, 8(8). doi: 10.1371/jour.

Suchman, L. et al. (2020) 'Bridging the Gap with a Gender Lens: How two Implementation Research Datasets were repurposed to inform Health Policy Reform in Kenya', Health Policy And Planning, 35(2), pp. 66-73. doi: 10.1093/heapol/czaa117.

Urquhart, R. et al. (2019) 'Factors influencing middle managers' commitment to the implementation of innovations in cancer care', Journal of Health Services Research and Policy. SAGE Publications Ltd, 24(2), pp. $91-99$. doi: 10.1177/1355819618804842.

VeneKlasen, L. and Miller, V. L. (2007) 'Power and Empowerment', in A New Weave of Power, People and Politics: the Action Guide for Advocacy and Citizen Participation. Practical Action Publishing.

Waithaka, D. et al. (2018) 'Describing and evaluating healthcare priority setting practices at the county level in Kenya', The International Journal of Health Planning and Management, 33(3), pp. e733-e750. doi: 10.1002/hpm.2527.

WHO (2010) Key components of a well functioning health system. Geneva. Available at: http://www.who.int/healthsystems/EN_HSSkeycomponents.pdf?ua=1 (Accessed: 16 July 2018).

\section{Supplementary Files}


This is a list of supplementary files associated with this preprint. Click to download.

- Supplementary1RehabManagerInterviewGuide.docx 